\title{
Superradiance in a Short-Pulse Free-Electron-Laser Oscillator
}

\author{
D. A. Jaroszynski \\ Department of Physics and Applied Physics, The University of Strathclyde, \\ John Anderson Building, 107 Rottenrow, Glasgow, G4 ONG, United Kingdom \\ P. Chaix \\ Commissariat à l'Énergie Atomique, BP12, 916680, Bruyères-le-Châtel, France \\ N. Piovella \\ Dipartimento di Fisica dell' Università, Via Celoria 16, 20133 Milano, Italy \\ D. Oepts, G. M. H. Knippels, A. F. G. van der Meer, and H. H. Weits \\ FOM-Instituut voor Plasmafysica “Rijnhuizen," Edisonbaan 14, 3439 MN Nieuwegein, The Netherlands
}

(Received 26 August 1996)

\begin{abstract}
We demonstrate that superradiance (SR) is a general feature of free-electron-laser (FEL) devices, incuding high gain amplifiers as well as low gain oscillators, and that SR pulses and spiking, due to synchrotron instabilities, have the same origin. In particular, we present a direct observation of SR from an FEL oscillator. The ultrashort SR pulses are characterized by a peak intensity and pulse duration which depend, respectively, on the square and the inverse square root of the electron current. The spectral brightness, $\mathcal{B}$, defined as the ratio between the efficiency and the relative rms spectral width, is constant and equal to 0.86. [S0031-9007(97)02481-2]
\end{abstract}

PACS numbers: 41.60.Cr, 42.50.Fx, 52.75.Ms

In 1954, Dicke [1] showed that the most efficient way of extracting energy from an inverted two-level atomic system is through cooperative emission where the radiation rate is enhanced by the mutual interaction of radiators through a common radiation field produced by neighboring emitters. The enhanced rate or superradiant (SR) rate is proportional to the number of emitters, $n_{e}$. Dicke showed that ultrashort pulses of coherent radiation are produced with a peak intensity scaling as $n_{e}^{2}$, and a temporal width scaling as $1 / n_{e}$. Recently, the same phenomenon has been predicted to occur in the high-gain free-electron laser (FEL) amplifier [2,3]. In the FEL the optical radiation field is produced by a process of Compton backscattering and bunching of the electrons on a radiation wavelength scale occurs in a ponderomotive potential due to the beat wave of the "undulator" wave and the counterpropagating laser wave $[4,5]$. More recently it has also been shown theoretically that superradiance can also occur in the FEL oscillator [6-8] and the collective atom recoil laser (CARL) [9] and has been linked to the postsideband $[10,11]$ or spiking regime [12,13]. As in Dicke SR, the mutual coupling between radiators through the electromagnetic field in the FEL is responsible for cooperative phenomena where the radiators do work against their own field and that produced by neighboring electrons. Collective radiation occurs as a result of the bunching of the electrons due to the mutually produced optical field. SR emission, as described in this Letter, starts from noise from a nonprebunched electron beam and, more importantly, occurs in the nonlinear stage of power evolution. SR should not be confused with coherent spontaneous emission from prebunched systems
[14]. In this Letter we demonstrate that superradiance is a general feature of FEL devices, incuding high gain amplifiers as well as low gain oscillators, and that superradiant pulses [15] and spiking [13] (due to synchrotron instabilities) have the same origin. In particular, we present direct observation of superradiance from an FEL oscillator. The experiments were carried out on the FELIX FEL located in The Netherlands [16].

We begin by showing that SR is a general phenomenon occurring in FEL oscillators. Consider a short electron pulse driven FEL with an electron bunch duration, $l_{b}$, less than the slippage length, $l_{s}=N_{u} \lambda$ [5], where $N_{u}$ is the number of undulator periods and $\lambda=\lambda_{u}\left(1+a_{u}^{2}\right) / 2 \gamma_{0}^{2}$, the resonance wavelength, where $\lambda_{u}$ and $a_{u}$ are the undulator period and rms parameter, respectively, and $\gamma_{0}$ is the initial electron energy in $m c^{2}$ units. It can be shown that at small signal levels the optical pulse shape will evolve into a steady state shape characterized by supermodes of the linear equations of motion and with a width $\sigma_{z}$ that depends on the cavity detuning, $\delta \mathcal{L}[7,8,17]$. A finite $\delta \mathcal{L}$ is necessary to maintain synchronism between electron and optical pulses in the cavity because of the effect of swept gain lethargy [18] which causes the optical pulse centroid to travel at a speed less than light in vacuum. The maximum gain occurs at $\delta \mathcal{L}=0.02 l_{b} g_{0}$ [7], where $g_{0}=N_{u}^{3} \gamma_{0}\left(I_{b} / I_{A}\right)\left(\lambda / z_{R}\right) f\left(a_{u}\right)^{2}$ is the continuous-wave (cw) small signal gain coefficient $[4,5], I_{b}$ is the peak current and $I_{A}=4 \pi \epsilon_{0} m c^{3} / e \sim 17 \mathrm{kA}$ is the Alfven limit current, $z_{R}$ is the Rayleigh length for the optical cavity and $f\left(a_{u}\right)=4 \pi\left[a_{u} /\left(1+a_{u}^{2}\right)\right]\left[J_{0}(\xi)-J_{1}(\xi)\right]$, with $\xi=$ $0.5 a_{u}^{2} /\left(1+a_{u}^{2}\right)$; the beam radius, $r_{b}$, has been assumed to 
match the beam waist radius, $\left(\lambda z_{R} / \pi\right)^{1 / 2}$. For smaller $\delta \mathcal{L}$ values the gain, which becomes independent of undulator length, drops to zero as $G=3^{3 / 2} l_{b}^{1 / 3} \delta \mathcal{L}^{2 / 3} / l_{c}$, where $l_{c}=\lambda / 4 \pi \rho$ is the cooperation length and $\rho=g_{0}^{1 / 3} /$ $\left(4 \pi N_{u}\right)$ is the FEL Pierce parameter [19]. Because the effect of lethargy is suppressed at saturation, the maximum efficiency occurs close to zero desynchronism, at $\delta \mathcal{L} \approx 0.18 l_{b}\left(\alpha_{0} l_{c} / l_{b}\right)^{3 / 2}$ [7], where $\alpha_{0}$ is the total cavity loss. The efficiency is $\eta \approx \rho \sqrt{l_{b} / \alpha_{0} l_{c}}$ and the peak power $\mathcal{P} \approx \rho P_{b}\left(l_{b} / \alpha_{0} l_{c}\right)^{2}$, where $P_{b}=m c^{2} \gamma_{0}\left(I_{b} / e\right)$ is the electron beam power. The superradiant character of the emission is expressed through $\mathcal{P} \propto Q^{2}$, where $Q=$ $I_{b}\left(l_{b} / c\right)$ is the charge, as obtained from the LandauGinzburg model of Refs. [7,8]. Similar results have been obtained for the high gain amplifier [20,21].

All the SR scaling and values for the SR parameters can be deduced from the condition that, at saturation, the optical pulse narrows until the electrons undergo an oscillation in phase during the interaction time limited by the optical pulse width, $\sigma_{z}[6]$. Strong saturation of the oscillator begins when the electrons execute half a synchrotron oscillation in the ponderomotive potential of dimensionless amplitude $|a|=\pi N_{u}^{2}\left(e \lambda|E| / \sqrt{2} m c^{2}\right) f\left(a_{u}\right)$, where $|E|$ is the peak electric field strength [5]. The field strength required to cause a half synchrotron oscillation is such that $|a| \approx \pi^{2}$. If the optical pulse width, $\sigma_{z}$, is less than the slippage length, $l_{s}$, the electrons will drift through the optical pulse and interact with it for a time $\tau=\sigma_{z} \mathcal{F} / c$, where $\mathcal{F}(\approx 2)$ is some fraction of the optical pulse duration depending on its shape. Hence the electrons interact with the optical pulse while traversing an effective number of undulator periods equal to $N_{s}=\mathcal{F} \sigma_{z} / \lambda$, instead of $N_{u}$ as in a cw FEL oscillator. Regrouping of the electrons while propagating in free space can give rise to small secondary pulses in an analogous way to "Burnham Chaio" ringing observed in Dicke SR systems [22]. The intracavity energy can be calculated from the electric field strength $|E|$, $\mathcal{F}_{\text {int }}=\sqrt{2 \pi} \sigma_{z} P_{\text {int }} / c=\sqrt{2 \pi} \sigma_{z} \epsilon_{0} \pi^{2} z_{R} \lambda|E|^{2} / 8$, where $P_{\text {int }}$ is the intracavity power and $\epsilon_{0}$ is the permittivity of free space. In the steady state, an oscillator will be in equilibrium with the gain, $\mathcal{G}$, equal to the loss, $\alpha_{0}$. The total power lost by the electrons, $\mathcal{E}_{o}$, on each passage is $\mathcal{E}_{o}=\alpha_{0} \mathcal{E}_{\text {int }}$ and the efficiency is $\eta=\alpha_{0} \mathcal{E}_{\text {int }} / \mathcal{E}_{e}$, where $\mathcal{E}_{e}=m c^{2} \gamma_{0}(Q / e)$ is the energy of the electron bunch. Assuming $\eta=|a|^{1 / 2} / 2 \pi N_{s} \approx 1 / 2 N_{s}$, a self-consistent value for $\sigma_{z}$ can be calculated to obtain the value $\sigma_{z} \approx$ $\left[\lambda / f\left(a_{u}\right) \mathcal{F}^{3 / 2}\right]\left(\alpha_{0} z_{R} / r_{c}\right)^{1 / 2}\left(m c^{2} / \mathcal{E}_{e}\right)^{1 / 2}$, where $r_{c}=$ $e^{2} / 4 \pi \epsilon_{0} m c^{2}$ is the classical radius of the electron. It should be noted that for single pulse propagation when the $l_{b}>l_{s}$ only electrons in the slippage region $l_{s}$ are involved in the interaction and therefore an effective electron bunch energy $\mathcal{E}_{e}=\mathcal{E}_{e}^{\prime} l_{s} / l_{b}$ with $l_{b}=c Q / \mathcal{I}_{b}$ should be used in the above calculations. The measurable efficiency, for the total electron bunch, is then $\eta^{\prime}=\eta l_{s} / l_{b}$. This is an essential step when calculating $\sigma_{z}$ for comparison with the experiment. All the relevant SR parameters can be expressed in terms of $\sigma_{z}$ giving the dependences on $\alpha_{0}$ and $I_{b}$ : efficiency, $\eta=1 / 2 N_{s} \propto 1 / \sigma_{z} \propto \sqrt{I_{b} / \alpha_{0}}$, optical energy, $\mathcal{E}_{o}=\eta \mathcal{E}_{e} \propto \mathcal{I}_{b}^{3 / 2} / \sqrt{\alpha_{0}}$, and peak power $\mathcal{P}=\mathcal{E}_{o} /\left(\sqrt{2 \pi} \sigma_{z}\right) \propto I_{b}^{2} / \alpha_{0}^{2}$.

We have observed SR in the FELIX FEL2 oscillator experiment [16]. FELIX operates in the large slippage regime and produces short optical pulses, as short as six cycles [23], for use by a varied user community [16]. FEL2 operates from 5 to $35 \mu \mathrm{m}$, and is driven by short electron bunches with $l_{b} \approx 1.5 \mathrm{~mm}$ and $Q$ up to $200 \mathrm{pC}$ from a $16-40 \mathrm{MeV} S$-band linac. The radiation is coupled out through a $2 \mathrm{~mm}$ hole in one of the cavity mirrors. The optical cavity has a Rayleigh length of $1.2 \mathrm{~m}$. The planar undulator consists of 38 periods of $6.5 \mathrm{~cm}$ and $a_{u}$ can be varied in the range 0 to 1.9 . In the experiments the duration of the optical pulses has been measured using a zerobackground second-order autocorrelation technique based on a nonlinear CdTe doubling crystal [23]. The overall resolution varies between $100 \mathrm{fs}$ at $5 \mu \mathrm{m}$, and $500 \mathrm{fs}$ at $35 \mu \mathrm{m}$. The efficiency of the FEL was determined from electron spectra measured using a fast, $0.22 \%$ resolution, electron spectrometer, placed after the FEL [24]. Optical spectra were measured with an evacuated grating spectrometer. Fast $\mathrm{HgCdTe}$ and pyroelectric detectors were used in conjunction with blocking filters to monitor the IR radiation. The cavity losses were determined from the measured exponential tail at the end of the optical macropulse.

To observe SR in the oscillator we have measured the efficiency and the optical pulse duration of the FEL as a function of $Q$ using the electron spectrometer and the autocorrelator, respectively. $Q$ was varied by adjusting the current from the electron gun to the linac while compensating for any energy decrease by increasing the rf power driving the accelerator. In this way we have varied $Q$ from 100 to $200 \mathrm{pC}$ while keeping the electron bunch length constant, the linac set at $39 \mathrm{MeV}$, and $a_{u}=1.53$ giving $\lambda=18.5 \mu \mathrm{m}$. For each value of $Q$ the oscillator's cavity detuning curves have been measured by varying $\delta \mathcal{L}$ to determine the maximum efficiency operating point. At low currents the electron macropulse was extended so as to observe saturation of the oscillator with the diminished gain. From the measurements of $\eta^{\prime}, \mathcal{E}_{e}$, and $\sigma_{z}$ the values of $\mathcal{P}$ and $\mathcal{E}_{o}$ have been determined. Optical pulse widths varying between $\sigma_{z}=75 \mu \mathrm{m}$ and $\sigma_{z}=110 \mu \mathrm{m}$ were measured. The measured autocorrelation curves were a close fit to an hyperbolic pulse shape, although at the highest powers measured, there was evidence of a tail in the curves consistent with the excitation of higher order supermodes [7,8] and evidence of Burnham Chaio ringing. Figure 1(a) shows $\mathcal{P}$ vs $Q^{2}$ for two different wavelengths clearly showing a square law dependence of the intensity on $Q$ confirming SR evolution in the FEL oscillator. The experimental data compare well with the calculated values, e.g., for $Q=200 \mathrm{pC}$ we expect $\mathcal{P}=264 \mathrm{MW}$ at $18.5 \mu \mathrm{m}$ for $\alpha_{0}=5.2 \%$ and $l_{b}=1.5 \mathrm{~mm}$; and $\mathcal{P}=$ $202 \mathrm{MW}$ at $8 \mu \mathrm{m}$ for $\alpha_{0}=7 \%$ and $l_{b}=1.3 \mathrm{~mm}$. In Fig. 1(b) we show the measured $\eta^{\prime}$ as a function of the 

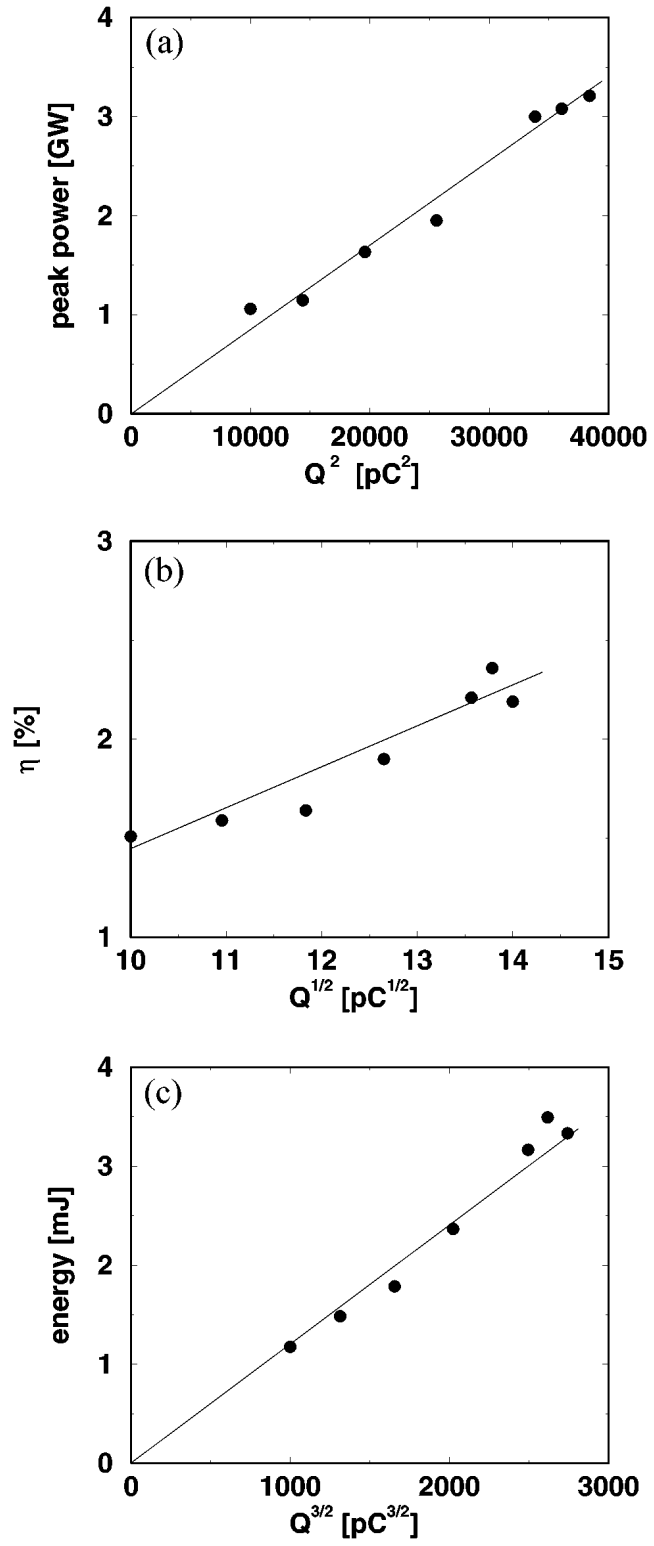

FIG. 1. (a) $\mathcal{P}_{\text {in }}$ vs $Q^{2}$, (b) $\eta^{\prime}$ vs $Q^{1 / 2}$, and (c) $\mathcal{F}_{\text {in }}$ vs $Q^{3 / 2}$,

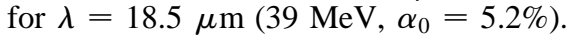

$\sqrt{Q}$ and in Fig. 1(c) we show the optical pulse energy lost from the cavity, $\mathcal{E}_{o}$, as a function of $Q^{3 / 2}$. The largest measured energy compares very well the predicted value of $180 \mu \mathrm{J}$. However, the actual efficiency, $\eta$, for electrons in a single slippage length is much larger than $\eta^{\prime}$ for the whole bunch. We calculate $\eta \approx 5.2 \%$ for $Q=200 \mathrm{pC}$. Efficiencies of more than $3.5 \%$ have been measured for $200 \mathrm{pC}$ and $30 \mathrm{MeV}$, at the longer wavelengths where slippage is strongest. Figure 2 shows the SR efficiency as a function of wavelength where $a_{u}$ has been varied from 0.73 to 1.58 while keeping the electron energy and charge constant at $30 \mathrm{MeV}$ and $200 \mathrm{pC}$, respectively. The predicted efficiencies calculated using the measured losses are also shown in Fig. 2. The high efficiency may account for the very large electron energy spreads observed in electron spectra. To measure the SR scaling on cavity loss,

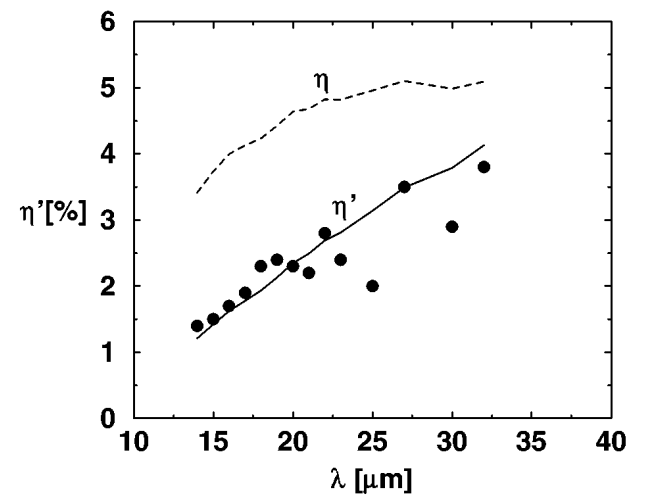

FIG. 2. Measured $\eta^{\prime}$ vs $\lambda$ by varying $a_{u}$ between 0.73 and 1.58. The solid line represents $\eta^{\prime}$ and the dotted line $\eta$ calculated using measured $\alpha_{0}$ values assuming $l_{b}=1.5 \mathrm{~mm}$.

$\alpha_{0}$ was varied between $5 \%$ and $15.5 \%$ by inserting very thin wires into the cavity. Figure 3 shows $\mathcal{P}$, derived from measurements of $\eta^{\prime}$ and $\sigma_{z}$, as a function of $1 / \alpha_{0}^{2}$ showing the SR scaling. Unfortunately, because of difficulties in carrying out the experiment, only three data points have been obtained. Finally, by measuring $\eta^{\prime}$ and the relative spectral bandwidth, $\sigma_{\lambda} / \lambda$, we have determined the relative brightness $\mathcal{B}=\eta /\left(\sigma_{\lambda} / \lambda\right)$ and have found it to be constant and close to $\sqrt{3} / 2$ as shown in Fig. 4. This provides the necessary link between short pulse FEL SR and the spiking regime that is characterized by $\mathcal{B}=\sqrt{3} / 2$ $[10,11]$. This is consistent with the Fourier transform products $2 \pi \sigma_{\nu} \sigma_{\tau} \approx 1$ observed and would indicate a frequency chirp in the optical pulse.

Finally, to elucidate the similarity between the high gain amplifier and the short pulse FEL oscillator, we consider an optical pulse propagating in the oscillator with $\delta \mathcal{L} \approx 0$ and $\alpha_{0} \ll 1$ where the FEL is still above threshold. By unfolding the oscillator cavity we can consider the optical pulse as effectively propagating in an amplifier operating in the exponential gain regime [25] with a cw electron beam and an infinitely long undulator. At larger values of $\delta \mathcal{L}$ care should be taken to consider an interaction region with a length $l_{s}$ copropagating with the optical pulse at a velocity $c\left(1-2 \delta \mathcal{L} / L_{u}\right)$, where $L_{u}$

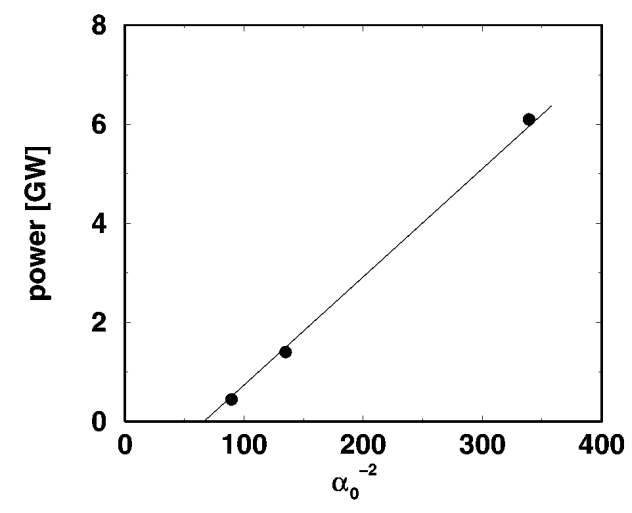

FIG. 3. $\mathcal{P}_{\text {in }}$ as a function of $\alpha_{0}^{-2}$. 


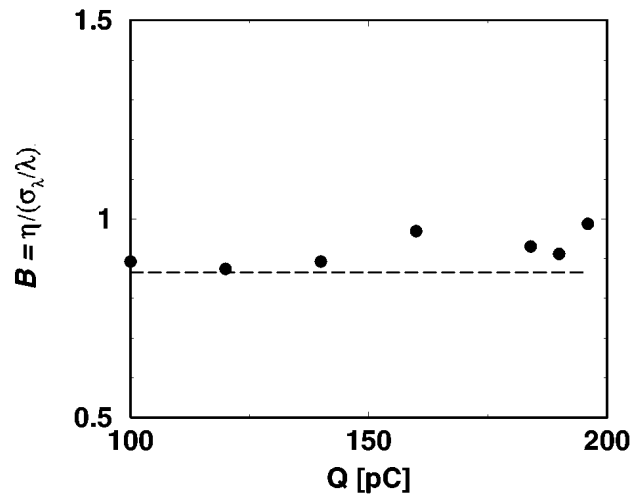

FIG. 4. $\mathcal{B}=\eta /\left(\sigma_{\lambda} / \lambda\right)$ showing a constant value of $\mathcal{B}=$ 0.86 where $l_{b}=1.5 \mathrm{~mm}$ in the estimate of $\eta$.

is the undulator length. In a SR FEL amplifier, the optical power of the SR pulse increases as it propagates over the electron beam with a peak power $\mathcal{P} \approx \rho P_{b}\left(l_{b}^{\prime} / l_{c}\right)^{2}$ and an efficiency $\eta \approx \rho \sqrt{l_{b}^{\prime} / l_{c}}$, where $l_{b}^{\prime}=l_{s}$ is the electron beam section swept by the SR pulse in $N_{u}$ undulator periods $[19,20]$. A SR oscillator, driven by electron pulses with length $l_{b}$ shorter than the slippage length, $l_{s}$, behaves as a SR amplifier in which the effective section of the beam swept by the optical pulse after $n$ passes through the cavity is $l_{b}^{\prime}=n l_{b}$. This can be possible only when the optical pulse duration is shorter than the slippage length, so that the electrons interact with the radiation for a number of undulator periods, $N_{u}$, determined by the width of the optical pulse. For small cavity detuning and small cavity loss, the SR radiation energy reaches a stationary condition when $n \approx 1 / \alpha_{0}$, so that the effective beam length in the amplifier analogy is $l_{b}^{\prime}=l_{b} / \alpha_{0}$. These conclusions are in agreement with the analytical results of Ref. [7], where the stationary solution of a Landau Ginzburg model for the fundamental supermode is derived, and of Ref. [8], where the transient evolution of SR pulses in FEL oscillators is analyzed for zero cavity detuning. From this discussion, we conclude that the SR saturated oscillator evolves exactly in the same way as the high gain amplifier under SR evolution.

In this Letter, we have demonstrated experimentally that the superradiant regime can be reached, studied, and used for optimal generation of radiation. Moreover, we have derived the main scaling laws for the superradiant regime of short pulse FEL's by analogy with the high gain amplifier and also with the assumption that the steady state optical pulse duration is a fraction of the synchrotron oscillation length. These results show that superradiance is not exclusive to high gain devices but can also occur in short pulse low gain systems. Actually superradiance is a quite general phenemenon occurring in a wide variety of devices and for very different choices of operating parameters. It is, for instance, now well established that high current long pulse FEL's produce spikes of radiation characterized by a constant spectral brightness $\mathcal{B} \approx 0.86$, where the typical length of spikes and the efficiency also scale as $Q^{-1 / 2}$ and $Q^{1 / 2}$, respectively. By measuring $\mathcal{B}$ we have shown that spiking and SR have the same general characteristics. Since superradiance is an optimum way to extract radiation from the electron beam, a number of issues still deserve attention, among them (i) cavity dumping may be an efficient way to obtain a high power FEL pulse, (ii) dynamic desynchronism may be used to combine large gain and large efficiency at saturation, and (iii) high power triggers secondary instabilities to limit cycle [26] and chaos, which may be stabilized.

[1] R. Dicke, Phys. Rev. 93, 99 (1954).

[2] R. Bonifacio and F. Casagrande, J. Opt. Soc. Am. B 2, 250 (1985).

[3] R. Bonifacio, B. W. J. McNeil, and P. Pierini, Phys. Rev. A 40, 4467 (1989).

[4] J. Madey, Nuovo Cimento Soc. Ital. Fis. B 50, 1 (1979).

[5] W. Colson, in Laser Handbook, edited by W. Colson, C. Pellegrini, and A. Renieri (North-Holland, Amsterdam, 1990), Vol. 6, p. 115.

[6] G. T. Moore and N. Piovella, IEEE J. Quantum Electron. 27, 2522 (1991).

[7] N. Piovella, P. Chaix, G. Shvets, and D. Jaroszynski, Phys. Rev. E 52, 5470 (1995).

[8] N. Piovella, Phys. Rev. E 51, 5147 (1995).

[9] R. Bonifacio, L. D. Salvo, L. Narducci, and E. D’Angelo, Phys. Rev. A 50, 1716 (1994).

[10] D. Iracane, P. Chaix, and J. Ferrer, Phys. Rev. E 49, 800 (1994).

[11] P. Chaix, D. Iracane, and C. Benoist, Phys. Rev. E 48, R3259 (1993).

[12] R. W. Warren, J. C. Goldstein, and B.E. Newnam, Nucl. Instrum. Methods Phys. Res., Sect. A 250, 19 (1986).

[13] R. W. Warren and J. C. Goldstein, Nucl. Instrum. Methods Phys. Res., Sect. A 272, 115 (1988).

[14] B. E. Newnam et al., SPIE, Proc. Soc. Photo-Opt. Instr., edited by C. A. Brau, S. F. Jacobs, and M. O. Scully, 453, 118 (1984).

[15] D. Oepts, A. van der Meer, and P. van Amersfoort, Infrared Phys. Technol. 36, 297 (1995).

[16] G. Dattoli and A. Renieri, in Laser Handbook, edited by M. Stitch and M. Bass (North-Holland, Amsterdam, 1985).

[17] F. Hopf, P. Meystre, and M. Scully, Phys. Rev. Lett. 35, 511 (1975).

[18] R. Bonifacio, C. Pellegrini, and L. Narducci, Opt. Commun. 50, 373 (1984).

[19] R. Bonifacio, L. D. S. Souza, P. Pierini, and N. Piovella, Nucl. Instrum. Methods Phys. Res., Sect. A 296, 358 (1990).

[20] R. Bonifacio et al., Riv. Nuova Cimento 15, 1 (1992).

[21] R. Bonifacio et al., Phys. Rev. Lett. 73, 70 (1994).

[22] D. Burnham and R. Chiao, Phys. Rev. 188, 667 (1969).

[23] G. M. H. Knippels et al., Phys. Rev. Lett. 75, 1755 (1995).

[24] W. A. Gillespie et al., Nucl. Instrum. Methods Phys. Res., Sect. A 358, 232 (1995).

[25] R. Bonifacio, N. Piovella, and B. W. J. McNeil, Phys. Rev. A 44, 3441 (1991).

[26] D. Jaroszynski et al., Phys. Rev. Lett. 70, 3412 (1993). 